\title{
On Some Ramsey Numbers for Quadrilaterals Versus Wheels
}

\author{
Janusz Dybizbański · Tomasz Dzido
}

Received: 24 January 2012 / Revised: 30 January 2013 / Published online: 28 February 2013

(C) The Author(s) 2013. This article is published with open access at Springerlink.com

\begin{abstract}
For given graphs $G_{1}$ and $G_{2}$, the Ramsey number $R\left(G_{1}, G_{2}\right)$ is the least integer $n$ such that every 2-coloring of the edges of $K_{n}$ contains a subgraph isomorphic to $G_{1}$ in the first color or a subgraph isomorphic to $G_{2}$ in the second color. Surahmat et al. proved that the Ramsey number $R\left(C_{4}, W_{n}\right) \leq n+\lceil(n-1) / 3\rceil$. By using asymptotic methods one can obtain the following property: $R\left(C_{4}, W_{n}\right) \leq n+\sqrt{n}+o(1)$. In this paper we show that in fact $R\left(C_{4}, W_{n}\right) \leq n+\sqrt{n-2}+1$ for $n \geq 11$. Moreover, by modification of the Erdős-Rényi graph we obtain an exact value $R\left(C_{4}, W_{q^{2}+1}\right)=q^{2}+q+1$ with $q \geq 4$ being a prime power. In addition, we provide exact values for Ramsey numbers $R\left(C_{4}, W_{n}\right)$ for $14 \leq n \leq 17$.
\end{abstract}

Keywords Ramsey numbers · Quadrilateral · Wheels

Mathematics Subject Classification (2000) $\quad 05 \mathrm{C} 55 \cdot 05 \mathrm{C} 15$

\section{Introduction}

In this paper all graphs considered are undirected, finite and contain neither loops nor multiple edges. Let $G$ be such a graph. The vertex set of $G$ is denoted by $V(G)$, the edge set of $G$ by $E(G)$, and the number of edges in $G$ by $e(G)$. Let $d(v)$ be the

This research was funded by the Polish National Science Centre (contract number DEC-2012/05/N/ST6/03063).

J. Dybizbański · T. Dzido $(\bowtie)$

Institute of Informatics, University of Gdańsk, Wita Stwosza 57, 80-952 Gdańsk Poland

e-mail: tdz@inf.ug.edu.pl

J. Dybizbański

e-mail: jdybiz@inf.ug.edu.pl 
degree of vertex $v$, and let $d_{1}(v)$ and $d_{2}(v)$ denote the number of the edges incident to $v$ colored with the first and the second color, respectively. By $\delta_{i}(G)$ we denote the minimum degree of $G$ in color $i$. The open neighborhood in color $i$ of vertex $v$ in graph $G$ is $N_{i}(v)=\{u \in V(G) \mid\{u, v\} \in E(G)$ and $\{u, v\}$ is colored with colori $\}$. Define $G[S]$ to be the subgraph of $G$ induced by the set of vertices $S \subset V(G)$. Let $P_{n}$ (resp. $C_{n}$ ) be the path (resp. cycle) on $n$ vertices. A wheel $W_{n}$ is a graph on $n$ vertices obtained from a $C_{n-1}$ by adding one vertex $w$ and making $w$ adjacent to all vertices of the $C_{n-1}$.

For given graphs $G_{1}, G_{2}$, the Ramsey number $R\left(G_{1}, G_{2}\right)$ is the smallest integer $n$ such that if we arbitrarily color the edges of the complete graph of order $n$ with 2 colors, then it always contains a monochromatic copy of $G_{1}$ colored with the first color or a monochromatic copy of $G_{2}$ colored with the second color. A coloring of the edges of $n$-vertex complete graph with 2 colors is called a $\left(G_{1}, G_{2} ; n\right)$-coloring if it does not contain a subgraph isomorphic to $G_{1}$ colored with the first color nor a subgraph isomorphic to $G_{2}$ colored with the second color.

The Turán number $t(n, G)$ is the maximum number of edges in any $n$-vertex graph which does not contain a subgraph isomorphic to $G$. A graph on $n$ vertices is said to be extremal with respect to $G$ if it does not contain a subgraph isomorphic to $G$ and has exactly $t(n, G)$ edges.

Some well known theorems will be used to prove the main result of this paper.

Theorem 1 (Ore [3]) Let $G$ be a graph on $n(n \geq 3)$ vertices. If $d(v)+d(w) \geq n$ for every pair of non-adjacent vertices $v$ and $w$ of $G$, then $G$ is Hamiltonian.

Theorem 2 (Rosta [7], Faudree and Schelp [2]) For all integers $n \geq 5$

$$
R\left(C_{4}, C_{n}\right)=\max \{n+1,7\} .
$$

Theorem 3 (Reiman [6]) For all integers $n \geq 4$

$$
t\left(n, C_{4}\right)<\frac{1}{4} n(1+\sqrt{4 n-3}) .
$$

Several results have been obtained for wheels and quadrilaterals. Surahmat et al. [8] showed that $R\left(C_{4}, W_{m}\right)=9,10$ and 9 for $m=4,5$ and 6 respectively. Independently, Kung-Kuen Tse [10] showed that $R\left(C_{4}, W_{m}\right)=10,9,10,9,11,12,13,14,16$ and 17 for $m=4,5,6,7,8,9,10,11,12$ and 13, respectively. In 2005, Surahmat et al. [9] obtained property that $R\left(C_{4}, W_{n}\right) \leq n+\lceil(n-1) / 3\rceil$. Suppose that we have an admissible coloring of $K_{m}$ without $C_{4}$ in color 1 and without $W_{n}$ in color 2 . Asymptotically we have a well-known property that $t\left(n, C_{4}\right) \approx \frac{1}{2} n^{\frac{3}{2}}$. Since $R\left(C_{4}, C_{n-1}\right)=n$ for $n \geq 7$, we obtain $\frac{1}{2} m(m-n) \approx \frac{1}{2} m^{\frac{3}{2}}$, which implies that $m-n \approx \sqrt{m}$ and $R\left(C_{4}, W_{n}\right)=n+\sqrt{n}+o(1)$. The main result of this work is the following.

Theorem 4 For all integers $n \geq 11$

$$
R\left(C_{4}, W_{n}\right) \leq n+\lfloor\sqrt{n-2}\rfloor+1
$$




\section{Main Theorem}

Proof (Theorem 4) For simplicity of notation, we set $k=\lfloor\sqrt{n-2}\rfloor$. Let us consider a graph $G=K_{n+k+1}$ and its decomposition $G=G_{1} \cup G_{2}$, where $V(G)=V\left(G_{1}\right)=$ $V\left(G_{2}\right)$ and $E\left(G_{i}\right)$ consists of all edges of $G$ in $i$ th color. Suppose that for graph $G$ there is a $\left(C_{4}, W_{n} ; n+k+1\right)$-coloring and let us consider such coloring.

First let us assume that there is a vertex $v \in V(G)$ such that $d_{1}(v) \leq k$. Then $d_{2}(v) \geq n$ and by $R\left(C_{4}, C_{n-1}\right)=n$ we immediately obtain a $W_{n}$ in the second color.

Now, suppose that $\delta_{1}(G) \geq k+2$. Let us consider integer $p$ such that $n \in\{(p-$ $\left.1)^{2}+2, \cdots, p^{2}+1\right\}$. Then $k=p-1$. Let $s=n-(p-1)^{2}$, one can see that $2 \leq s \leq 2 p$. In this case the minimum possible number of edges in color 1 in $G$ is

$$
\begin{gathered}
\left\lceil\frac{1}{2}(n+k+1) \delta_{1}(G)\right\rceil \geq \frac{1}{4}(n+k+1)(2 p+2) \geq \\
\geq \frac{1}{4}(n+k+1)\left(1+\sqrt{4\left(p^{2}+p+1\right)-3}\right) \geq \\
\geq \frac{1}{4}(n+k+1)\left(1+\sqrt{4\left(p^{2}-p+1+s\right)-3}\right) \geq \\
\geq \frac{1}{4}(n+k+1)(1+\sqrt{4(n+k+1)-3})>t\left(n+k+1, C_{4}\right),
\end{gathered}
$$

a contradiction.

The last case to consider is $\delta_{1}(G)=k+1$. In this case $G_{1}$ has at most $t(n+k+$ $\left.1, C_{4}\right)=\left\lceil\frac{(n+k+1) \delta_{1}(G)}{2}\right\rceil+A$ edges. Similarly to the previous case let us consider integer $p$ such that $n \in\left\{(p-1)^{2}+2, \cdots, p^{2}+1\right\}$. Then $k+1=p$. Let us take vertex $v \in V(G)$ such that $d_{1}(v)=k+1$, subgraph $G^{\prime}=G_{2}\left[N_{2}(v)\right]$ and two vertices $v_{1}, v_{2} \in V\left(G^{\prime}\right)$, where the edge $\left\{v_{1}, v_{2}\right\} \in E\left(G_{1}\right)$. Then $\left|V\left(G^{\prime}\right)\right|=n-1$ and in subgraph $G^{\prime}$ we have $d_{2}\left(v_{1}\right)+d_{2}\left(v_{2}\right)=2(n-2)-\left(d_{1}\left(v_{1}\right)+d_{1}\left(v_{2}\right)\right)$. We have the following

Claim $d_{1}\left(v_{1}\right)+d_{1}\left(v_{2}\right) \leq 2 \delta_{1}(G)+A$ or $d_{1}\left(v_{1}\right)+d_{1}\left(v_{2}\right) \leq 2 \delta_{1}(G)+A+1$ depending on the parity of $\delta_{1}(G)$ and $(n+k+1)$.

Proof If $\delta_{1}(G)$ and $|V(G)|=(n+k+1)$ are odd, then it is impossible that for all vertices $w \in V(G)$ we have $d_{1}(w)=\delta_{1}(G)$. In the worst situation, when all $A$ edges are adjacent to $v_{1}$ or $v_{2}$, we have that $d_{1}\left(v_{1}\right)+d_{1}\left(v_{2}\right) \leq 2 \delta_{1}(G)+A+1$.

We will prove that $d_{2}\left(v_{1}\right)+d_{2}\left(v_{2}\right) \geq n-1$ for all vertices $v_{1}, v_{2} \in V\left(G^{\prime}\right)$ such that $\left\{v_{1}, v_{2}\right\} \in E\left(G_{1}\right)$. In this case we obtain a contradiction because by Ore's Theorem subgraph $G^{\prime}$ contains a $C_{n-1}$ and $G$ contains a $W_{n}$ in the second color.

The remaining part of the proof is divided into three parts. 
Table 1 Values needed to prove that $d_{2}\left(v_{1}\right)+d_{2}\left(v_{2}\right) \geq n-1$ for $11 \leq n \leq 17$

\begin{tabular}{lrrrrrrr}
\hline$n$ & 11 & 12 & 13 & 14 & 15 & 16 & 17 \\
\hline$|V(G)|=n+k+1$ & 15 & 16 & 17 & 18 & 19 & 20 & 21 \\
$n-1$ & 10 & 11 & 12 & 13 & 14 & 15 & 16 \\
$t\left(|V(G)|, C_{4}\right)$ & 30 & 33 & 36 & 39 & 42 & 46 & 50 \\
$A$ & 0 & 1 & 2 & 3 & 4 & 6 & 8 \\
$d_{2}\left(v_{1}\right)+d_{2}\left(v_{2}\right) \geq$ & 10 & 11 & 12 & 13 & 14 & 14 & 14 \\
\hline
\end{tabular}

Table 2 Values needed to prove that $d_{2}\left(v_{1}\right)+d_{2}\left(v_{2}\right) \geq n-1$ for $18 \leq n \leq 26$

\begin{tabular}{|c|c|c|c|c|c|c|c|c|c|}
\hline$n$ & 18 & 19 & 20 & 21 & 22 & 23 & 24 & 25 & 26 \\
\hline$|V(G)|=n+k+1$ & 23 & 24 & 25 & 26 & 27 & 28 & 29 & 30 & 31 \\
\hline$n-1$ & 17 & 18 & 19 & 20 & 21 & 22 & 23 & 24 & 25 \\
\hline$t\left(|V(G)|, C_{4}\right)$ & 56 & 59 & 63 & 67 & 71 & 76 & 80 & 85 & 90 \\
\hline$A$ & - & - & 0 & 2 & 3 & 6 & 7 & 10 & 12 \\
\hline$d_{2}\left(v_{1}\right)+d_{2}\left(v_{2}\right) \geq$ & 21 & 24 & 25 & 26 & 26 & 26 & 26 & 26 & 25 \\
\hline
\end{tabular}

\section{1. $11 \leq n \leq 17$}

In this case $\delta_{1}(G)=p=4$. The exact values of $t\left(n, C_{4}\right)$ are known for all $n \leq 21$, see [1]. In addition, this paper covers all extremal graphs. Table 1 contains all values needed to prove the inequality $d_{2}\left(v_{1}\right)+d_{2}\left(v_{2}\right) \geq n-1$.

One can see that for all $11 \leq n \leq 15$ the proof is complete. For case $n=16$ let us consider the graph $G_{1}$. If it is the only extremal graph for $t\left(20, C_{4}\right)[1]$ then its maximum degree is 5 , so by Ore's Theorem $G^{\prime}$ contains a $C_{15}$ and $G$ contains a $W_{16}$ in the second color. If $\left|E\left(G_{1}\right)\right| \leq 45$, then $A \leq 5$ and $d_{2}\left(v_{1}\right)+d_{2}\left(v_{2}\right) \geq 15$. By similar considerations in case $n=17$, if $G_{1}$ is the only extremal graph for $t\left(21, C_{4}\right)[1]$ then $G^{\prime}$ contains a $C_{16}$ and $G$ contains a $W_{17}$. If $\left|E\left(G_{1}\right)\right|=49$ and there exists a vertex $w \in V(G)$ such that $d_{1}(w)=8$, then we obtain a $C_{4}$ in color 1 in $G$ (consider $\delta_{1}(G)=4$ and all possible edges in color 1 from $N_{1}(w)$ to the remaining vertices of $G$ ). If $d_{1}(w) \leq 7$ for all vertices $w \in V(G)$, then by Ore's Theorem $G^{\prime}$ contains a $C_{16}$ and $G$ contains a $W_{17}$. Then $A \leq 6$ and $d_{2}\left(v_{1}\right)+d_{2}\left(v_{2}\right) \geq 16$ and we are done.

2. $18 \leq n \leq 26$ In this case $\delta_{1}(G)=p=5$. The exact values and extremal graphs for $t\left(n, C_{4}\right)$ are known for all $22 \leq n \leq 31$, see [11]. Table 2 presents all values needed to finish the checking the inequality $d_{2}\left(v_{1}\right)+d_{2}\left(v_{2}\right) \geq n-1$ for $18 \leq n \leq 26$. We will mark with ${ }^{\prime}-^{\prime}$ the case when $A<0$.

3. $n \geq 27$

In this case $p \geq 6$. We have that in $G^{\prime} d_{1}\left(v_{1}\right)+d_{1}\left(v_{2}\right) \leq 2 \delta_{1}(G)+1+A$, then in $G^{\prime} d_{2}\left(v_{1}\right)+d_{2}\left(v_{2}\right) \geq 2(n-2)-\left(2 \delta_{1}(G)+1+A\right)=2 n-2 p-5-A$. In order to finish the proof we have to show that $2 n-2 p-5-A \geq n-1$, i.e. $A \leq n-2 p-4$. Observe that $w(n, p)=t\left(n+p, C_{4}\right)-\left\lceil\frac{(n+p) p}{2}\right\rceil \leq$ $\frac{1}{4}(n+p)(1+\sqrt{4(n+p)-3})-\left\lceil\frac{(n+p) p}{2}\right\rceil$ is an increasing function of $n$, i.e. $w\left(n_{1}, p\right)>w\left(n_{2}, p\right)$ if $n_{1}>n_{2}$. Then, the maximal possible value of $A$ holds for $n=p^{2}+1$. For even $p$ we have that $t\left(n+p, C_{4}\right) \leq \frac{\left(p^{2}+p+1\right)(p+1)}{2}-\frac{1}{2}$ and 
$\left\lceil\frac{(n+p) p}{2}\right\rceil=\frac{\left(p^{2}+p+1\right) p}{2}$. For odd $p$ we have that $t\left(n+p, C_{4}\right) \leq \frac{\left(p^{2}+p+1\right)(p+1)}{2}$ and $\left\lceil\frac{(n+p) p}{2}\right\rceil=\frac{\left(p^{2}+p+1\right) p}{2}+\frac{1}{2}$. In both situations we obtain that $A \leq \frac{p^{2}+p}{2}$ and for all $p \geq 6, A \leq p^{2}-2 p-3$.

Taking $n=q^{2}+1$ in Theorem 4, we have

Corollary 5 For all integers $q, q \geq 4$

$$
R\left(C_{4}, W_{q^{2}+1}\right) \leq q^{2}+q+1 .
$$

\section{Erdős-Rényi Graph}

Let $q$ be a prime power. The famous Erdős-Rényi graph $E R(q)$, first constructed by Erdős and Rényi in 1962, was studied in detail by Parsons in [4]. We know the following properties of $E R(q)$ :

- ER $(q)$ has $q^{2}+q+1$ vertices, $q+1$ vertices with degree $q$ and $q^{2}$ vertices with degree $q+1$

- $E R(q)$ does not contain a subgraph $C_{4}$

- in $E R(q)$ there are no two adjacent vertices of degree $q$

- in $E R(q)$ no vertex of degree $q$ belongs to a subgraph $K_{3}$

Let $H(q)$ denote the subgraph of $E R(q)$ obtained by deleting one vertex of degree $q$. By the third property of $E R(q)$, the subgraph $H(q)$ contains $2 q$ vertices with degree $q$ and $q^{2}-q$ vertices with degree $q+1$. One can observe that for all vertices $w$, the degree $d(w)$ in the complement of $H(q)$ is at most $q^{2}-1$. By this fact, the complement of $H(q)$ does not contain a $W_{q^{2}+1}$, so there exists a $\left(C_{4}, W_{q^{2}+1} ; q^{2}+q\right)$-coloring. By this fact and by Corollary 5 we have the following

Theorem 6 For $q \geq 4$ being a prime power

$$
R\left(C_{4}, W_{q^{2}+1}\right)=q^{2}+q+1 .
$$

\section{Exact Values for Small Wheels}

Up to date values for $R\left(C_{4}, W_{n}\right)$ are known only for $n \leq 13$. We determined the next four values as follows:

Theorem 7 1. $R\left(C_{4}, W_{14}\right)=18$,

2. $R\left(C_{4}, W_{15}\right)=19$,

3. $R\left(C_{4}, W_{16}\right)=20$,

4. $R\left(C_{4}, W_{17}\right)=21$.

Proof By Theorem 6 we immediately obtain $R\left(C_{4}, W_{17}\right)=21$. In order to determine an upper bound for all remaining cases we use Theorem 4 . For a lower bound we present appropriate matrix of critical coloring (see Fig. 1). These matrices were obtained by using simulated annealing to find $C_{4}$-free graphs with a minimum degree 4 . 


$\mathrm{X} 1111100000000000$
$1 \mathrm{X} 100011000000000$
$11 \mathrm{X} 00000110000000$
$100 \mathrm{X} 1000001100000$
$1001 \mathrm{X} 000000011000$
$10000 \mathrm{X} 00000000111$
$010000 \mathrm{X} 1001000100$
$0100001 \mathrm{X} 000100010$
$00100000 \mathrm{X} 01010010$
$001000000 \mathrm{X} 0101001$
$0001001010 \mathrm{X} 000100$
$00010001010 \mathrm{X} 00010$
$000010001000 \mathrm{X} 1010$
$0000100001001 \mathrm{X} 001$
$00000110001000 \mathrm{X} 01$
$000001011001100 \mathrm{X} 0$
$0000010001000110 \mathrm{X}$
$\left(C_{4}, W_{14} ; 17\right)$-coloring

$\mathrm{X} 11111000000000000$
$1 \mathrm{X} 1000110000000000$
$11 \mathrm{X} 000001100000000$
$100 \mathrm{X} 10000011000000$
$1001 \mathrm{X} 0000000110000$
$10000 \mathrm{X} 000000001110$
$010000 \mathrm{X} 10010001000$
$0100001 \mathrm{X} 0000100001$
$00100000 \mathrm{X} 010010101$
$001000000 \mathrm{X} 01100010$
$0001001010 \mathrm{X} 0000100$
$00010000010 \mathrm{X} 000011$
000010010100X00100
0000100010000X1001
00000110000001X010
000001001010100X00
0000010001010010X0
00000001100101000X
$\left(C_{4}, W_{15} ; 18\right)$-coloring

\begin{abstract}
X111110000000000000
$1 \mathrm{X} 10001110000000000$

11X0000001100000000

100X100000011000000

1001X00000000110000

10000X0000000001110

010000X100010001000

0100001X00000000101

01000000X0001100010

001000000X010100001

0010000000X01010100

00010010010X1001000

000100001011X000000

0000100011000X00100

00001000001000X0011

000001100001000X010

0000010100100100X00

00000100100000110X1

000000010100001001X

$\left(C_{4}, W_{16} ; 19\right)$-coloring
\end{abstract}

Fig. 1 Lower bound for $R\left(C_{4}, W_{n}\right), 14 \leq n \leq 16$

Open Access This article is distributed under the terms of the Creative Commons Attribution License

which permits any use, distribution, and reproduction in any medium, provided the original author(s) and the source are credited.

\title{
References
}

1. Clapham, CRJ, Flockhart, A, Sheehan, J.: Graphs without four-cycles. J. Graph Theory 13, 2947 (1989) 
2. Faudree, RJ, Schelp, RH.: All Ramsey numbers for cycles in graphs. Discret. Math. 8, 313-329 (1974)

3. Ore Ø.: Note on Hamilton circuits. American Mathematical Monthly 67(1), 55 (1960)

4. Parsons, TD.: Graphs from projective planes. Aequationes Math. 14, 167-189 (1976)

5. Radziszowski, SP.: Small Ramsey Numbers, Electron. J. Combin., Dynamic Survey 1, revision \#13, http://www.combinatorics.org (2011)

6. Reiman I.: Uber ein Problem von K. Zarankiewicz, Acta Math. Acad. Sci. Hungar., 9, 269-279 (1958)

7. Rosta, V.: On a Ramsey Type Problem of J.A. Bondy and P. Erdös, I \& II. J. Combin. Theory Series B 15, 94-120 (1973)

8. Surahmat, Baskoro, ET., Nababan SM., The Ramsey numbers for a cycle of length four versus a small wheel, Proceedings of the 11th Conference Indonesian Mathematics 172-178, Malang, Indonesia, July 22-25 (2002)

9. Surahmat, Baskoro, ET., Uttunggadewa, S., Broersma HJ., (2005) An upper bound for the Ramsey number of a cycle of length four versus wheels, LNCS 3330, Springer, Berlin, 181-184

10. Kung-Kuen, T.: On the Ramsey number of the quadrilaterals versus the book and the wheel. Aust. J. Combin. 27, 163-167 (2003)

11. Yuansheng, Y, Rowlison, P.: On extremal graphs without four-cycles. Utilitas Math. 41, 204-210(1992) 